\title{
A CHARACTERIZATION OF A SPACE WITH COUNTABLE INFINITY
}

\author{
AKIHIRO OKUYAMA
}

Abstract. It is well known that, for a countable discrete space $N$,

$$
|\beta N-N|=2^{2 N_{0}} \text {. }
$$

So, any completely regular $T_{1}$ space $X$ with $|\beta X-X| \leqq \boldsymbol{N}_{0}$ does not contain any infinite discrete subspace. In this paper, we characterize those completely regular $T_{1}$ spaces with countable infinity as follows: Such a space $X$ is characterized by the two properties.

(a) $X$ is pseudocompact.

(b) There exist a compact metric space $Y$ and a continuous $\operatorname{map} f$ from $X$ onto $Y$ so that the subset $Y_{0}=\left\{y: f^{-1}(y)\right.$ is not compact $\}$ of $Y$ is countable and $\operatorname{cl}_{\beta X} f^{-1}(y)-f^{-1}(y)$ is one point whenever $y \in Y_{0}$. (In particular, for any $y$ in $Y_{0}, \beta\left(f^{-1}(y)\right)-f^{-1}(y)$ is one point if $X$ is normal.)

1. Introduction. Let $\beta X$ denote the Stone-Cech compactification of a completely regular $T_{1}$ space $X$, let $|A|$ denote the cardinal number of a set $A$, and finally, let us call the set $\beta X-X$ the infinity. It is well known that $|\beta N-N|=2^{2^{\aleph_{0}}}$ for the countable discrete space $N$ (cf. [1]). Thus, in general, $|\beta X-X|$ is large. But, on the other hand, there exist spaces with the infinity consisting of only one point (for example, the space of all countable ordinal numbers with usual topology), and if we form the product of such a space with a compact $T_{1}$ space consisting of countably many points, we obtain a space with the infinity consisting of countably many points (for brevity, we say countable infinity).

A completely regular $T_{1}$ space with one-point infinity has been characterized in $[1,6 \mathrm{~J}]$ and a locally compact, regular $T_{1}$ space with finite or countable compactification (that is, a space with finite or countable infinity for some compactification) has been discussed and characterized in [3] and [4].

The purpose of this paper is to characterize a completely regular $T_{1}$ space with countable infinity through the use of a suitable compact metric space and a continuous map. Also, a completely regular $T_{1}$ space with finite infinity is characterized as a special case.

Received by the editors June 23, 1970.

AMS 1969 subject classifications. Primary 5453.

Key words and phrases. Stone-Čech compactification, countable infinity, compact metric space, pseudocompact space. 
TheOREM $1 .{ }^{1}$ For each completely regular $T_{1}$ space $X$, the following conditions are equivalent:

(1) $|\beta X-X| \leqq \aleph_{0}$,

(2) $X$ is pseudocompact and there exists a continuous map from $X$ onto a compact metric space $Y$ so that the subset

$$
Y_{0}=\left\{y \in Y: f^{-1}(y) \text { is not compact }\right\}
$$

is countable and $\left|\mathrm{cl}_{\beta X} f^{-1}(y)-f^{-1}(y)\right|=1$ whenever $y \in Y_{0}$. (In particular, $\left|\beta\left(f^{-1}(y)\right)-f^{-1}(y)\right|=1$ for $y \in Y_{0}$ if $X$ is normal.)

(3) For any continuous map $f$ from $X$ onto an arbitrary compact metric space $Z$ the subset

$$
Z_{0}=\left\{z \in Z: f^{-1}(z) \text { is not compact }\right\}
$$

of $Z$ is countable and $\left|\operatorname{cl}_{\beta X} f^{-1}(y)-f^{-1}(y)\right| \leqq \aleph_{0}$ whenever $z \in Z_{0}$. (In particular, $\left|\beta\left(f^{-1}(z)\right)-f^{-1}(z)\right| \leqq \aleph_{0}$ for $z \in Z_{0}$ if $X$ is normal.)

As a special case, for a space with finite infinity we have Theorem 2 .

TheOREM 2. For each completely regular $T_{1}$ space $X$ the following conditions are equivalent:

(1) $|\beta X-X|=n$ (a positive integer).

(2) $X$ is pseudocompact and there exists a continuous map from $X$ onto a compact metric space $Y$ so that the subset $Y_{0}=\left\{y \in Y: f^{-1}(y)\right.$ is not compact $\}$ consists of just $n$ points and $\left|\operatorname{cl}_{\beta X} f^{-1}(y)-f^{-1}(y)\right|=1$ whenever $y \in Y_{0}$. (In particular, $\left|\beta\left(f^{-1}(y)\right)-f^{-1}(y)\right|=1$ for $y \in Y_{0}$ if $X$ is normal.)

The proofs that (1) and (2) are equivalent in Theorems 1 and 2 are similar and therefore we only prove Theorem 1 .

2. Proof of Theorem 1. (1) implies (2). First, the condition (1) implies the pseudocompactness of $X$. Because, if $X$ were not pseudocompact, it would contain a closed subset $F$ of $X$ which could be saturated with a countable (infinite) discrete subspace $N$ of the real line $R$ by a continuous function $f: X \rightarrow R$; that is, $f(F)=N$ and $f^{-1}(N)$ $=F$. Then we have $\beta(f)^{-1}\left(\operatorname{cl}_{\beta R} N-N\right) \subset \beta X-X$, where $\beta(f)$ denotes the extension of $f$ over $\beta X$. Since $\beta N=\operatorname{cl}_{\beta R} N$ holds (cf. [1]) and $N$ has an uncountable infinity, $\beta X-X$ must be uncountable, which contradicts to the condition (1).

Next, let us put $\beta X-X=\left\{p_{1}, p_{2}, \cdots\right\}$, where $p_{i} \neq p_{j}$ for any $i \neq j$. Since $\beta X$ is a completely regular $T_{1}$ space, for any distinct $m, n$

${ }^{1}$ The author sincerely thanks the referee for suggesting the present form of Theorem 1 which is slightly stronger than the original version. The author is also indebted to the referee for suggesting an elegant proof for Theorem 1. 
there exists a continuous map $f_{m n}$ from $\beta X$ into $I$ (the unit closed interval) with values $f_{m n}\left(p_{m}\right)=0$ and $f_{m n}\left(p_{n}\right)=1$. Define

$$
e: \beta X \rightarrow P=\prod_{m, n} I \quad \text { (a metric space) }
$$

by $(e(x))_{m, n}=f_{m n}(x)$, and put $f=e \mid X$ and $Y=f(X)$. Then $Y$ is a compact metric space. Moreover, $Y=e(\beta X)$. This follows from the fact that the continuous image of a pseudocompact space is pseudocompact, any pseudocompact subspace of a metric space is compact (and therefore closed) and $Y$ is also dense in $e(\beta X)$. Furthermore, since $X$ is pseudocompact and each point of $Y$ is $G_{\delta}, f$ is a $Z$-map and hence a $W Z$-map; i.e., $\operatorname{cl}_{\beta X}\left(f^{-1}(y)\right)=e^{-1}(y)$ for each $y \in Y$ (cf. [2]). Let us put $q_{i}=e\left(p_{i}\right)$ for $i=1,2, \cdots$. Then for any $y \in Y$ which is distinct from any $q_{i}$ we have $e^{-1}(y) \subset X$ and so $f^{-1}(y)=e^{-1}(y)$ is compact. For $q_{i}$, from the definition of $e$, we can easily see that $q_{i}$ 's are mutually distinct and so we have $e^{-1}\left(q_{i}\right)=f^{-1}\left(q_{i}\right) \cup\left\{p_{i}\right\}$. This completes the proof in the case that $X$ is a completely regular $T_{1}$ space. If $X$ is normal, we can assert $\beta\left(f^{-1}\left(q_{i}\right)\right)=\operatorname{cl}_{\beta X} f^{-1}\left(q_{i}\right)$ for $i=1,2, \cdots$. Thus the proof is completed.

(2) implies (1). Let $X, Y, f$ and $Y_{0}$ be given as in (2). By the same reasoning as before, we have $\operatorname{cl}_{\beta X}\left(f^{-1}(y)\right)=\beta(f)^{-1}(y)$ for each $y \in Y$. For each $y_{i} \in Y_{0}$ we denote $\operatorname{cl}_{\beta X} f^{-1}\left(y_{i}\right)=f^{-1}\left(y_{i}\right) \cup\left\{p_{i}\right\}$ for some $p_{i} \in \beta X-X$. Of course, $\beta X$ is the complete inverse image of $Y$ by $\beta(f)$ and therefore we have $\beta X=U\left\{\beta(f)^{-1}(y): y \in Y\right\}$ $=\bigcup\left\{\operatorname{cl}_{\beta X} f^{-1}(y): y \in Y\right\}=\bigcup\left\{f^{-1}(y): y \in Y\right\} \cup\left\{p_{1}, p_{2}, \cdots\right\}$. This completes the proof.

(1) is equivalent to (3). The proof is almost clear. For the direction $(1) \Rightarrow(3)$, if we put $\beta X=X \cup\left\{p_{1}, p_{2}, \cdots\right\}$, then $Z_{0}=\left\{\beta(f)\left(p_{i}\right)\right.$ : $i=1,2, \cdots\}$ satisfies the required conditions. (Notice that again $f$ is a $W Z$-map.) For the direction $(3) \Rightarrow(1)$, it suffices to let $Z$ be a one-point space.

\section{REFERENCES}

1. L. Gillman and M. Jerison, Rings of continuous functions, University Series in Higher Math., Van Nostrand, Princeton, N. J., 1960. MR 22 \#6994.

2. T. Isiwata, Mappings and spaces, Pacific J. Math. 20 (1967), 455-480; correction, ibid. 23 (1967), 630-631. MR 36 \#2127.

3. K. D. Magill, Jr., N-point compactifications, Amer. Math. Monthly 72 (1965), 1075-1081. MR 32 \#3036.

4. - Countable compactifications, Canad. J. Math. 18 (1966), 616-620. MR 33 \#6578.

University of Pittsburgh, Pittsburgh, Pennsylvania 15213

Osaka University of Education, Osaka, Japan 